\title{
MISCELÁNEA
}

\section{Reseña de Gestión del cuidado interprofesional/ de Dr. Ricardo Ayala Valenzuela, Editor}

\author{
Interprofessional Care Management Review / by Dr. Ricardo Ayala Valenzuela, Editor
}

\section{Revisão da Gestão de Cuidados Interprofissionais/ Dr. Ricardo Ayala Valenzuela, Editor Lorena Paola Bettancourt-Ortega}

Escuela de Enfermería. Universidad de Valparaíso. Chile

Académica Adjunto del Departamento del adulto y adulto Mayor de la Escuela de Enfermería de la Universidad de Valparaíso, Directora de Magister en Enfermería de la misma Unidad.

Cómo citar esta reseña en edición digital: Bettancourt-Ortega, L.P. (2020). Reseña de Gestión del cuidado interprofesional/ de Dr. Ricardo Ayala Valenzuela, Editor. Cultura de los Cuidados

(Edición digital), 24(57). Recuperado de http://dx.doi.org/10.14198/cuid.2020.57.23

Correspondencia: Escuela de Enfermería. Universidad de Valparaíso. Chile

Correo electrónico: lorena.bettancourt@uv.cl

\begin{abstract}
By reading this book you will find he views of different professions regarding the management of care and its evolution, about its influence on the nursing sector and how this responsibility for caring over time has been exercised. The author provides a complementary vision to the management of care, an issue that remains in constant tension and politicization in Chile. In this direction, he gives us his own vision and that of various authors in the field of health who, in a fragmented way, describe multiple visions of the subject: from the state and its legislation, from the actors, the same professionals who travel from the collaboration to the dispute of the field of
\end{abstract}

action which is the health management. Then it presents as necessary not only the theoretical construction of the concept of "interprofessionality", but the need to insert it into the educational system of future health professionals, involving it in all areas of professional work. Hence, it proposes a new look, betting on new organizational models in the future, which will not be exempt from analysis and subsequent debate by stakeholders.

Keywords: Health management, health workers, nursing.

\section{RESUMEN}

Al leer este libro se encontrarán los puntos de vista de diferentes profesiones respecto a la gestión del cuidado y su 
evolución, sobre su influencia en el estamento enfermería y cómo se ha ejercido esta responsabilidad de cuidar en el tiempo. El autor aporta una visión complementaria a la gestión del cuidado, cuestión que se mantiene en permanente tensión $\mathrm{y}$ politización en Chile. En esta dirección, nos entrega la visión propia y la de diversos autores del campo de la salud que de forma fragmentada van describiendo múltiples visiones que del tema se tienen: desde el estado y su legislación, desde los actores, los mismos profesionales que transitan desde la colaboración a la disputa del campo de acción cual es la gestión de la salud. Luego presenta como necesario no solo la construcción teórica del concepto de "interprofesionalidad", sino que la necesidad de insertarlo dentro del sistema educativo de los futuros profesionales de la salud, involucrándola en todos los ámbitos del trabajo profesional. De allí que propone una nueva mirada, apostando por nuevos modelos organizacionales a futuro, que no estarán exentos de análisis y posterior debate por parte de los interesados.

Palabras clave: Gestión en salud; trabajadores de la salud; enfermería. Fuente Bireme Desc.
Ao ler este livro, você encontrará as opiniões de diferentes profissões sobre o gerenciamento do cuidado e sua evolução, sobre sua influência no setor de enfermagem e como essa responsabilidade pelo cuidado ao longo do tempo foi exercida. O autor fornece uma visão complementar à gestão do cuidado, questão que permanece em constante tensão e politização no Chile. Nesse sentido, ele nos dá sua própria visão e a de vários autores da área da saúde que, de maneira fragmentada, descrevem múltiplas visões do sujeito: do estado e de sua legislação, dos atores, os mesmos profissionais que viajam de a colaboração para a disputa do campo de ação que é a gestão da saúde. A seguir, apresenta-se necessária não apenas a construção teórica do conceito de "interprofissionalidade", mas a necessidade de inseri-lo no sistema educacional dos futuros profissionais de saúde, envolvendo-o em todas as áreas do trabalho profissional. Por isso, propõe um novo visual, apostando em novos modelos organizacionais no futuro, que não estarão isentos de análises e subsequentes debates das partes interessadas.

Palavras-chave : Gestão em saúde; trabalhadores da saúde; Enfermagem.

\section{RESUMO}

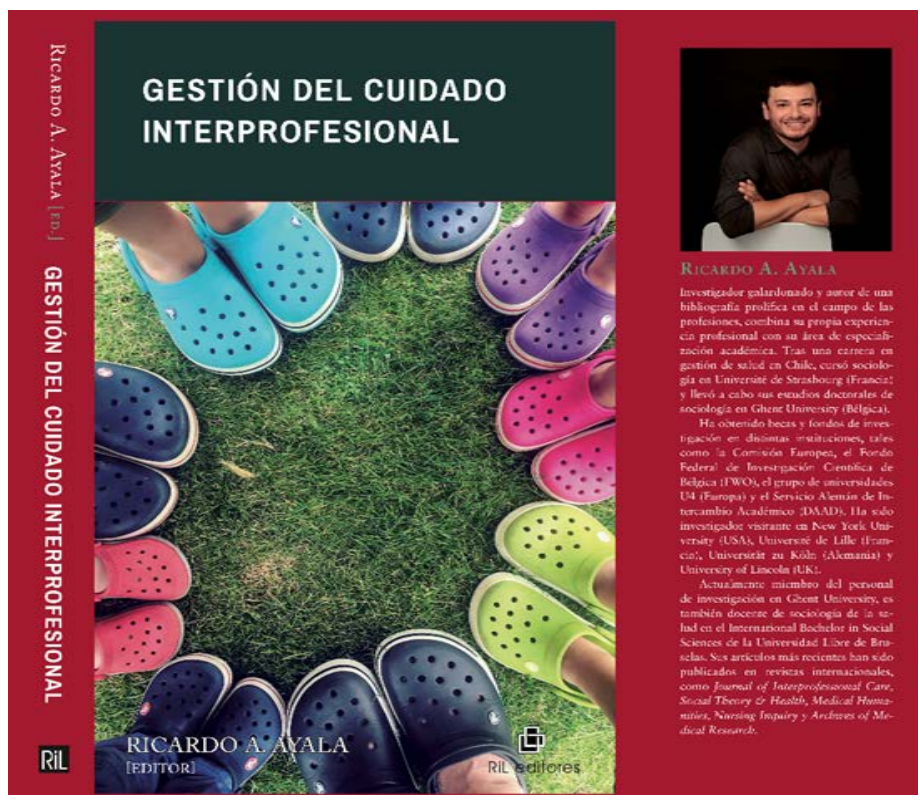


Antes de presentar este libro creo necesario poner en contexto algunas cuestiones que quizá para el futuro lector, estudiantes, profesionales de enfermería, gestoras del cuidado, investigadores en la línea, puedan ser relevantes a la hora de leer (y tomar posición) sobre este texto. La enfermería en Chile está atravesando un período crítico, se conjugan en ello un cambio en la institucionalidad, con la revisión del libro $\mathrm{V}$ del código sanitario, que le da sustento legal a la profesión enfermera y a otras disciplinas de salud que han ido evolucionando en el tiempo, cambiando como se relacionan entre si y como se acomodan según el contexto sociopolítico.

Se aprecian diferencias sustanciales en la calidad de la enseñanza de la enfermería, la gran cantidad de estudiantes que egresan anualmente superan con creces los cargos disponibles en el mercado laboral, lo que ha hecho que el empleo de los jóvenes se precarice cada vez más. En el contexto internacional, se declaró el 2020 como el año de la enfermera, movimientos globales como Nursing Now, amparados por la OMS buscan darle más visibilidad y protagonismo a este profesional de la mano de temáticas como la especialización en enfermería, la participación en los espacios de decisión, donde las enfermeras pueden aportar a la gestión interprofesional a través del trabajo colaborativo. Ligado a ello, el nombramiento reciente de la Directora Nacional de enfermería fue recibido con gran entusiasmo por el gremio enfermero.
Estamos ante una época de cambios, donde a las enfermeras se nos ha dado un rol descrito legalmente que hemos asumido, pero al mismo tiempo, las otras profesiones en salud exigen participar del cuidado, varias de ellas afirman que cuidan. Además, otras profesiones de ámbito social también desarrollan miradas más macro de cómo los determinantes sociales han influido en el cuidado de la salud de la población y como pueden contribuir al mejoramiento de las condiciones de vida de las comunidades.

Al leer este libro se encontrarán los puntos de vista de diferentes profesiones respecto a la gestión del cuidado y su evolución, sobre su influencia en el estamento enfermería y cómo se ha ejercido esta responsabilidad de cuidar en el tiempo. El autor aporta una visión complementaria a la gestión del cuidado, cuestión que se mantiene en permanente tensión $\mathrm{y}$ politización en Chile. En esta dirección, nos entrega la visión propia y la de diversos autores del campo de la salud que de forma fragmentada van describiendo múltiples visiones que del tema se tienen: desde el estado y su legislación, desde los actores, los mismos profesionales que transitan desde la colaboración a la disputa del campo de acción cual es la gestión de la salud. Luego presenta como necesario no solo la construcción teórica del concepto de "interprofesionalidad", sino que la necesidad de insertarlo dentro del sistema educativo de los futuros profesionales de la salud, involucrándola en todos los ámbitos del 
Revista científica de la Asociación de Historia y Antropología de los Cuidados (Universidad de Alicante)

trabajo profesional. De allí que propone una nueva mirada, apostando por nuevos modelos organizacionales a futuro, que no estarán exentos de análisis y posterior debate por parte de los interesados.

El escrito se organiza en dos partes, Transformación e Integración:

En la primera parte: Transformación, Osvaldo Artaza en su: "Cuidado médico y la gestión de enfermería: La historia de una reforma", nos presenta una descripción de los cambios en el ámbito del cuidado asistencial y de la gestión de enfermería a partir de la Reforma de Salud, así como los cambios sociales que han afectado la construcción de la identidad profesional y la relación interprofesional de médicos y enfermeras. Artaza presenta algunos factores que permitirían la comprensión global del mundo del trabajo en el área de la salud como son el género y la participación laboral de las mujeres, la horizontalidad de las relaciones de trabajo y el empoderamiento de las enfermeras y usuarios. Por último, el autor plantea su visión a futuro de cómo se organizará la atención de salud y cuáles serán sus principales características.

En el segundo capítulo, Juán Rapimán, en su "Transformación de la experticia del técnico de enfermería", muestra una detallada descripción histórica de la trayectoria del personal de apoyo, desde los que no contaban con ninguna capacitación en los inicios, hasta los técnicos de nivel superior. A mi modo de ver, esto es lo más valioso del capítulo, cómo ha sido el devenir de este grupo que, al compás de los cambios en la salud de chile, se esfuerza por ir evolucionando y tomando las responsabilidades en atención clínica que le competen. También aquí queda de manifiesto la expectativa de que el gremio sea reconocido en las responsabilidades que le ha tocado desempeñar y en el futuro de las aspiraciones que tienen.

$$
\text { En el capítulo } 3 \text { "Terapia }
$$

Ocupacional: profesión que cuida, acompaña e incluye socialmente ante la discapacidad", Laura Rueda nos muestra su visión de cuidado como acompañamiento al desempeño ocupacional de las personas durante todo el desarrollo vital. Trata de describir el papel de la profesión en sus cincuenta años de trayectoria, la función de los terapeutas, los ámbitos de acción y desde donde se posicionan conceptualmente para brindar cuidado, desde su punto de vista, con un fuerte base en la ética del cuidar.

En el capítulo 4 "Trabajo social e interdisciplinariedad de la gestión del cuidado: descolonización del poder en el ámbito de la salud" de Luis Vivero Arriagada, el análisis, desde el pensamiento crítico, aborda las dinámicas que se producen en el campo de la salud referida a las relaciones de poder entre y hacia los diferentes actores que participan en la gestión del cuidado. Afirma que existe una forma hegemónica de conocer y validar el conocimiento y que es a través de ello como 
se ejerce el poder y se mantiene la división social del trabajo en su forma piramidal. Para lograr prácticas más democráticas e interdisciplinarias, afirma que debemos respetar y dar espacio a los diferentes saberes, incluyendo a las ciencias sociales.

En el capítulo 5 "Gestión del cuidado de la salud sexual y reproductiva de la mujer: enfoque de curso de vida, sensibilidad de género y ética", Lorena Binfa comienza haciendo un recorrido histórico de la profesión de matrona, desde la partera de la época colonial, pasando por las aulas universitarias, hasta nuestros días, donde describe cómo se ha tomado bajo su responsabilidad la salud sexual y reproductiva de los chilenos a través de todo el ciclo vital. Además, fundamenta el desarrollo disciplinar, haciendo un análisis de cómo es necesario retomar temas urgentes de la salud de la mujer como son los relacionados a la esfera sociocultural y de los determinantes sociales, en una perspectiva más amplia que el rol tradicional de la matrona.

En el capítulo 6, "Cuidado social e intervención social directa ¿Superposición o trabajo interprofesional?", Sandra Iturrieta nos proporciona una visión muy interesante de los procesos de expansión y cierre de las profesiones vinculadas al campo de la salud , planteando cómo podrían variar las posibilidades de empleo, las condiciones del mercado laboral y las relaciones entre las profesiones, con dos posibilidades: la desaparición de las profesiones como las conocemos o, al contrario, la transformación que sufrirán para adaptarse a la re conceptualización del trabajo, a la superposición de roles y a su influencia sobre la gestión del cuidado.

En la segunda parte del libro, "Integración”, el capítulo 7: "Cuidado interprofesional: legislación e integración", de Bernardo Alarcón, plantea una interesante discusión sobre la gestión del cuidado como cuerpo legislativo exclusivo para la profesión enfermera y muestra algunas limitaciones teóricas a la hora de delimitar campos de competencia y autonomía en las diferentes profesiones que ejecutan roles y tareas de gestión. Aborda el cuidado interprofesional como posibilidad jurídica. Esta esfera de actuación delimitada por ley, la práctica, ha sido más difícil de delimitar por las diversas formas de hacer que involucra, con un lenguaje genérico que se adaptaría a la evolución profesional, a la realidad social cambiante, sin necesidad de cambios legislativos en el tiempo. Describe también como las enfermeras en la defensa de su campo han recurrido históricamente al argumento legal como herramienta para resolver los conflictos, en desmedro de la actuación profesional o el dialogo directo.

En el capítulo 8, "Más allá del trabajo en equipo: Nuevas perspectivas de colaboración interprofesional", de Ricardo Ayala, editor de la obra, nos encontramos con un análisis comparativo entre el concepto de trabajo en equipo como una de las formas primordiales de definición del 
Revista científica de la Asociación de Historia y Antropología de los Cuidados (Universidad de Alicante)

trabajo en enfermería que ha perdurado en el tiempo, centrado en la rutina y en la fragmentación de los roles que reproduce jerarquías, entre otros, con el trabajo colaborativo, visto como una transformación cultural que ofrece una alternativa para movilizar racionalmente los recursos para cuidar. Además, identifica cuales son las barreras, entre ellas: la construcción de una institucionalidad propia de las disciplinas y su validación, la construcción de la identidad profesional lo que redundaría en la segregación y las posibles formas en que se pueden superar como la construcción de un modelo colaborativo interprofesional que, a juicio del autor, lograría disminuir los errores clínicos, disminuir la duplicación de esfuerzos, en definitiva, potenciar la calidad del cuidado a través de la comunicación de los equipos. Este modelo de socialización debería comenzar por enseñarse desde el pregrado para desarrollar desde el inicio la identidad interprofesional en paralelo a su identidad mono profesional.

En el Capítulo 9, "El cuidado interprofesional como tema y sistema", Tomas Koch entrega elementos teóricos para analizar la gestión interprofesional del cuidado. Como primer punto analiza a las organizaciones de salud bajo la teoría de sistemas, bajo la perspectiva de Luhmann, que ve a las profesiones como sistemas autónomos que interactúan y se distinguen de otros a través precisamente de tal o cual distinción respecto de reglas de inclusión, roles o reglamentos. Gestión interprofesional entonces se constituiría como la interacción de varios sistemas profesionales que establecen contacto a través de la comunicación (diálogo, apertura y flexibilidad) y la investigación interprofesional, para desde ahí avanzar hacia experiencias de colaboración que permitan mecanismos de gestión interprofesional.

A modo de conclusión:

En primer lugar, como enfermera y, en segundo lugar, como académica de una Escuela de Enfermería de una Universidad Pública, este libro me parece relevante porque permite conocer los diversos puntos de vista de los profesionales, que, en mayor o menor medida, han tenido o tienen actualmente experiencia en el tema. En cierta forma se establece un dialogo indirecto que puede abrir nuevos espacios de discusión. Quedarse solo en la visión interna de la situación es peligroso a futuro, por cuanto no contribuye a enriquecer el conocimiento sobre los fenómenos.

Las enfermeras y otros gremios de la salud no podemos quedarnos estancadas en posturas rígidas que no aportan realmente a la discusión de lo que ha sido la gestión del cuidado, sin preguntarnos por las fortalezas e inconvenientes que se presentan para su aplicación práctica, o por las acciones que deberemos emprender para mejorar en conjunto la situación de salud de los chilenos, que es en definitiva nuestro objetivo común. 
Se ha producido, a mi modo de ver, un proceso de contracción de la disciplina enfermera, en cierto modo porque de ser una disciplina plenipotenciaria en el cuidado de las personas ha visto su quehacer invadido por otras profesiones más nuevas, más focalizadas que han profundizado en sus marcos teóricos y en sus quehaceres, llenando de alguna manera el vacío dejado por las enfermeras en algún sentido. Por otro lado, hay un proceso de expansión de estas últimas que en algún sentido está determinado por la falta de definición legal de su ámbito de acción que les permitiría explorar nuevos rumbos, en este último punto creo que han legitimado su quehacer a través de la práctica y las enfermeras hemos dejado este desarrollo de la práctica para ampararnos en lo legal. La Enfermería por su parte ha migrado fuertemente hacia la gestión, alejándose del cuidado directo de las personas a su cargo.

La segunda parte del libro propone como se podría instaurar un esquema de trabajo interprofesional que permita una transformación de los sistemas de salud. En ese sentido, podría cambiarse o modificarse el foco en cada disciplina y migrar a resolver las problemáticas de las personas atendidas, de manera integral. Muchas veces los ámbitos de acción profesional son reducidos, no toman todos los aspectos que debieran y entonces los pacientes quedan en una desprotección, en una falta de servicios que no satisface la necesidad de salud.
¿Y qué le falta al libro? ¡Sugiero obviamente la incorporación de una enfermera! Una profesional con vasta experiencia en la gestión del cuidado, que haya sido testigo y partícipe en el tiempo de la Reforma de salud, sus fortalezas y debilidades, las ganancias en el tiempo, los cambios en las relaciones de poder entre las profesiones involucradas, el estilo de trabajo $y$, por qué no, la visión que ella tenga de cómo las otras profesiones se han empoderado del cuidado, sus puntos de vista y el manejo desde dentro del cuidado de la salud de la población.

$$
\text { ¿Entonces, como }
$$

empezamos el cambio? En el ámbito individual:

A mi modo de ver, las enfermeras transitamos de un modelo disciplinar a uno interdisciplinar, no es un cambio dicotómico. Ud. fortalezca su disciplina, estudie, base su práctica en teorías y modelos propios, en la evidencia, en la investigación, hágase partícipe y usuaria del propio conocimiento enfermero para que pueda exponer y fundamentar cambios. Sea consciente de lo que saben las enfermeras, de lo que hacen y de lo que podrían llegar a ser; solo así se podrá posicionar en equipos multiprofesionales, será respetada y tendrá la última palabra en lo que al cuidado se refiere, pero tendrá claridad de lo que hacen los otros profesionales y cómo todos juntos pueden llegar a la meta de cuidar. No necesitará ampararse en la ley, porque también estará legitimada en la práctica. 
Revista científica de la Asociación de Historia y Antropología de los Cuidados (Universidad de Alicante)

En el ámbito institucional:

Las instituciones universitarias deben enseñar el modelo interdisciplinar, en la teoría y en la práctica, en hospitales clínico docentes donde los estudiantes trabajen juntos desde el inicio de sus carreras, donde todas las disciplinas sean respetadas y tengan el mismo peso, sin que ninguna tenga el monopolio. Cambiemos el modelo de trabajo en equipo, por trabajo colaborativo, que permitiría entonces a las enfermeras tener una identidad propia, pero a la vez una identidad interprofesional, cuando trabajamos interdependientemente. Al investigar en gestión interprofesional, podría avanzarse en definiciones más consistentes del campo ocupacional y tomar el campo para investigar lo que está sucediendo, desarrollar la línea de investigación de gestión del cuidado de manera más interprofesional, que es lo que se está pidiendo en todos los fondos concursables en el país. Una solución que podría contribuir al problema sería formar centros de estudio, que son campos de aplicación multidisciplinar donde confluyen aquellos que están involucrados en problema, con una mirada más amplia, relevante que está fuera de la disciplina como ideología.

En relación al cuerpo legislativo que nos rige: Revisitar la noción de la gestión del cuidado, darle contenido a la definición para evitar que sigan existiendo los conflictos de roles, superposición, abandono, disputa y problemas de interpretación de la ley. Por último, no debe verse esta publicación como una crítica directa, sino como una oportunidad de visualizar cómo han evolucionado las profesiones, cómo se ven a sí mismas, de qué manera pueden contribuir al cuidado, su apertura a formas de colaboración interprofesional y cómo ven a la enfermería en este mismo punto. Entonces, no hay que tenerle miedo a la visión externa de la profesión, entre ellas habrá valiosas consultas, dudas, visiones y sugerencias que harán de la contribución la riqueza y sistematización del método. 